\title{
Bridges as City Landmarks: A Critical Review on Iconic Structures
}

\author{
Ayca Arslan \\ Uşak University, Faculty of Architecture and Design, Uşak, Turkey
}

Received: September 21th 2020, Revised: November 9th 2020, Accepted: November 15th 2020

Refer: Arslan, A., (2020), Bridges as City Landmarks: A Critical Review on Iconic Structures, Journal of Design Studio, V.2, N.2, pp 85-99,

A. Arslan ORCID: 0000-0002-5262-1429

DOI: $10.46474 /$ jds.798072 https://doi.org/10.46474/jds.798072

\begin{abstract}
This paper investigates bridges from 'landmark of cities' point of view under aesthetics theme. In fact, bridges gave many samples at history with high aesthetic qualities; constructed with diverse materials and vernacular traditional construction methods of the region and era they had been built. Bridges, however, known as engineering products, today it is expected from them to accommodate high aesthetic qualities as being perceptual productions of their environment. It is this 'construction and aesthetics' combination that makes them landmarks, which will be demonstrated in the paper by selected cases include both high structural and aesthetical qualities, transforms them to landmarks and icons of their city. On the other hand, by the development of new materials and construction styles, aesthetic consideration come forward by productions of sculptural steel construction bridges in cities. Basically paper consists of two parts, which first one is literature review that introduces aesthetic values in design, landmark features, classifications of iconic concept and $2^{\text {nd }}$ part includes general mapping on bridges from past to present with different functional and constructional styles. In the article, aesthetics in design and landmark concept have been introduced strongly in order to understand the meaning of 'icon'. Thus especially the design content of Enzio Manzini have been introduced in the article with his work '21.th Century Values of Design' which he explains the importance of aesthetics in design. In addition, at this part, design elements as an architectural language formed by 'point, line, plane' and visual elements such as; 'shape, form, color and texture' have been introduced. In order to continue, design principles such as rhythm, balance, emphasis, scale \& proportion, hierarchy have been introduced in the study to reach landmark concept. At second part approximately fifteen unique bridge design and construction have been investigated from all around world such as; Florence, Singapore, London, Sydney, San Francisco, France, Amsterdam, China, Australia and Turkey. All of these selected bridges accommodate iconic features uniquely and differently from each other which transform them to a landmark of their city or environment. The uniqueness of the bridges depends on superiority of some features such as; the length, the height, type, function, construction, material, form, referring the construction system design such as; helix, space frame, steel tensegrity, cable stayed steel swing bridge, single arch and hangers, pods steel high tech materials, steel, pylons and abutments, suspension bridge, steel through arch bridge, wave form made up of seven undulating curved steel, stone build, a three stage pointed arched stone bridge. As indicated, all construction systems are unique and in addition they show highly aesthetic criteria. Thus, when a unique structure meets with technology and/or high aesthetic qualities such as design principles and color, the structure of a building becomes an icon for its city. Then, at $3^{\text {rd }}$ part, four iconic bridge cases have been investigated through their aesthetic and landmark values. At the end, important keys will be collected for aesthetically designed future bridge constructions. To sum up, in the article, it is aimed to reveal landmark concept in the cities over bridge cases which are very aesthetical and flexible structures by their forms, construction styles, materials and functional diversities.
\end{abstract}

Keywords: Landmarks, icons, aesthetics, innovation.

\section{Introduction}

This paper titled as 'Bridges as Landmarks', investigates bridge structures from past to present with an architectural aesthetical value. However, bridges are known as engineering products, it's the aesthetical value that comes forward by the urban spatiality they create. The aim of the paper is to indicate the importance of aesthetic values in bridge design especially from urban spatiality features and landmark 
criteria. Study makes a wide investigation on selected iconic bridge cases from past to present such as; Sinan's Büyük Çekmece Bridge in Turkey, Golden Gate Bridge in U.S, Millau Viaduct in France and Zaragoza bridge in Spain. Cases have been selected due to their iconic features which make them landmarks in their environment.

As the part of literature review, general mapping on bridges have been achieved around the world within a search through superiority features such as ; too long, too high, too aesthetic \&artistic, too multi-functional and/or too high-tech by construction techniques which compromise the limitation of the 15 sample case selections. On the other hand, bridges have been investigated under two themes; structural and functional variations of bridge structures from past to present. Firstly; structural superiority and diversity introduces bridges with different construction styles such as; stone-made, steel, cable-stayed, steel space frame structures. These structural analyses revealed how technology and architecture have an important role on changing bridge designs. Then, with second theme as functional diversity, bridges have been investigated and classified from past to present through their functions such as; railroad bridges, pedestrian \& bike bridges, and lastly as a superiority feature; bridges as buildings with enclosed spaces such as shops, exhibition areas where one can both travel in the museum and cross the river.

\section{Problem Statement \& Field study}

Problem: When analyzed from past to recent day, it is found that bridges include diverse aesthetic qualities not only being functional structures, but also the creation of urban spatial imagination which transforms them landmarks inside the city.
"In this respect; study tries to find answers to the question; "1. For to be a landmark, can aesthetic values of a bridge come forward than structural value?"

Field Study: 4 bridge constructions that include iconic architecture features: Büyük Çekmece Bridge, Golden-Gate Bridge, Milla Viaduct, and Zaragoza Bridge have been analyzed deeply by parameters that are determined by 15 sample case analyses.

Aims \& objectives: The aim of the paper is to reveal and demonstrate the importance of aesthetic values on bridge designs which transform them landmarks in urban spatiality.

Methodology: The study includes both quantitative and qualitative research methods. a comprehensive literature review introduces; theoretical review about aesthetic values in design, landmark concept for iconic values in design and basic design principles for artistic values. By making a general mapping on bridges from past to present, super adjectives have been determined such as; too high, too long, too aesthetic, too functional (multifunctional) and too technological by construction methods, which transforms a simple bridge structure to a landmark and 15 unique bridge designs have been selected for sample analyses to determine features in design to be an 'icon' that have been used at basic case analyses. Deep case analyses have been achieved on selected 4 bridges, by parameters that have been determined both at first and second parts. which create high quality landmark criteria. Lastly at fourth part, conclusions have been given with important keys for bridge designs, aesthetic values and landmark criteria for to give a light for future studies on bridge designs (Table 1.). 
Table 1. Methodology of the study

\begin{tabular}{|l|l|l|}
\hline 1.LITERATURE REVIEW & SAMPLE CASE ANALYSES & BASIC CASE ANALYSES \\
\hline $\begin{array}{l}\text { 1)Aesthetic values in design } \\
\text { 2)Landmark concept }\end{array}$ & $\begin{array}{l}\text { 15 landmark bridge analyses } \\
\text { 3)Basic design elements and } \\
\text { to determine parameters for } \\
\text { landmark and icon criteria }\end{array}$ & $\begin{array}{l}\text { 4 iconic bridge analyses by } \\
\text { parameters that are } \\
\text { determined by } 15 \text { sample case } \\
\text { analyses }\end{array}$ \\
\hline
\end{tabular}

\section{Literature Review and General Mapping}

\subsection{Aesthetic Values, Landmark Criteria and Principles of Design}

This part introduces literature review as three part theoretical architectural investigations on; aesthetic values, landmark criteria and design principles and elements that have been used for analyzing cases by aesthetic urban spatiality.

\subsubsection{Aesthetic Values of Design:}

Design has been defined by different theorists till now. There are some descriptions for word 'design' which comes from old days. For example, Booker (1964) describes design like 'Simulating what we want to make before we make it as many times as may be necessary to feel confident in the final result.' And at fine arts, design is the creative period that covers the study of sketches and plans for a project. As Enzio Manzini indicates; 'design will have to re-examine the very basis of its culture and he puts particular emphasis on the significance of the role of values in designing' (Inns T, 2007: 306).

In addition, Manzini asserts; 'the need for an aesthetic to be based upon a value system, and emphasizes the important role that an aesthetic of sustainability would play in the transition to a new economic and social order' (Inns T, 2007: 307).

As Enzio Manzini in the book 'Designing for the 21.th Centuries-Values of Design', indicated that (2010); 'Every era has its own ethics and aesthetics. Aesthetics represents the way in which a historical period and the values it contains 'take form'. During the first part of the century, design played a decisive role in giving form to modernity. During the 1980's, the better or worse, design was the protagonist of an aestheticization of things: an aestheticization which, on the whole, has proven incapable of countering the more generalized aesthetic decay for the world. Today, the perspective of sustainable society has not yet 'taken form' and an aesthetic of sustainability have yet to be born' (Inns T, 2007).

The theme of aesthetics must be considered seriously: as it become commonplace to view the aesthetic dimension as secondary; an extra to be added when the rest has been resolved, a luxury for those who have everything. In reality aesthetics is connected to ethics in the sense that no true, profound aesthetic renewal can occur without being based on a value system (Buchanan R., Doordan D., Margolin V., 2010).

\subsubsection{Landmark Value analyses}

"Do quantitative and qualitative characteristics of size, innovation, beauty, location and surrounding, simplicity, complexity, historical traces, longevity make a bridge iconic?"

As the subject of the paper; 'Bridges as Landmarks', it's better to start by meaning of icon; An icon is an image as defined by Oxford English Dictionary (2011), it can be a statue, a figure, representation or a portrait. A representation of some sacred personage honored with a warship or regarded as secret (Inns T, 2007).

Through C.W. Morris's definitions of icon, the basic aspect of an icon is 'aesthetic' and an aesthetic sign is defined as an iconic sign (an image) whose design datum is a value. Iconic, in generally refers to events, people and /or

Journal of Design Studio, v:2 n:2 
objects that are famous for those within the fields in question (notably popular culture, fashion and sport) and often also for the public at large and have special symbolic/aesthetic significance attached to them and the key factors of iconic are such as; unique design, concept or symbolic values of design construction made it iconic. In addition, as Charles Jencks indicated; 'The new type of architecture has emerged in the last decade: the iconic landmark building, which challenges the traditional architectural monument. In the past, public buildings expressed shared meaning through well-known conventions. Today those conventions are superseded by commercial forces and the quest for instant fame. Public architecture is now required to be an amazing piece of surreal sculpture as well as something that appeals to a diverse audience-at once provocative and practical yet without the context that religion and ideology once provided' (Jencks C,2000). Here, Jencks emphasizes how iconic buildings became landmarks especially at last decades and compromises with past as traditional monumental landmarks such as Eiffel Tower which is also landmark too. Today by the help of changing technology iconic landmark buildings have been created such as; Gehry's Guggenheim Museum at Bilbao. Each year many tourists visit Bilbao to see landmark as museum, which demonstrates the power of landmarks in a city. Of course Bilbao effect has precedents, but the Eiffel Tower was built for no compelling purpose except to be an icon, later it came to symbolize Paris (Jencks C,2000).

\subsubsection{Design Principles:}

In architectural language, basic design principles that are formed by; 'point, line, plane' and visual elements such as; 'shape, form, color and texture' are commonly used during creating visual compositions. Visual language composes of these elements and their relationships. Conceptual elements become visual elements when they take form, size, color and texture. On the other hand, when these conceptual elements have been organized by design principles, artistic compositions are determined. This part introduces some basic design principles such as; rhythm, balance, scale \& proportion and hierarchy. (Kaymakcan M, 2006)

a. Rhythm Principle: A unifying movement characterized by a patterned repetition or alteration of formal elements or motifs in the same or modified form. (Ching,1996)

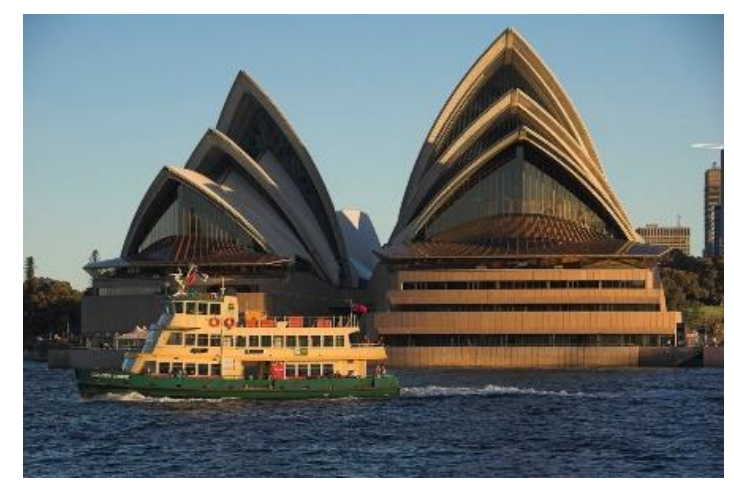

Figure. 1.Sidney Opera House, John Utzon, 19571973(source: https://www.archdaily.com/65218/adclassics-sydney-opera-house)

b. Symmetry: The balanced distribution and arrangement of equivalent forms and spaces on opposite sides of a dividing line or plane, or about a center or axis (Ching,1996) (Fig.2).

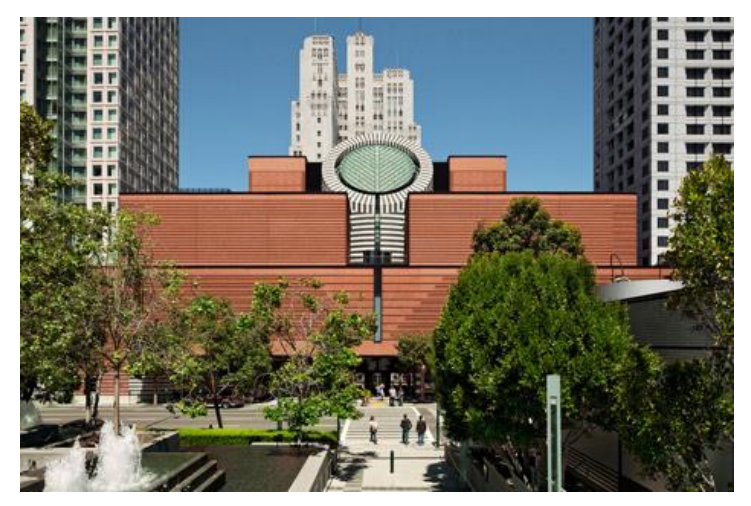

Figure.2. Maria Botta, San Francisco Museum of Modern Art, (source:

https://www.dezeen.com/2015/08/10/postmodernis m-architecture-sfmoma-san-francisco-museum-ofmodern-art-mario-botta-snohetta-craig-dykersextension/)

c. Scale \& Proportion Principle: While proportion pertains to an ordered set of 
mathematical relationships among the dimensions of a form or a space, scale refers to how we perceive or judge the size of something in relation to something else. In detailing with the issue of scale, therefore, we are always comparing one thing to another (Ching,1996: 162).

d. Hierarchy Principle: The principle of hierarchy implies that in most if not all architectural compositions, real differences exist among their forms and spaces. These differences reflect the degree of importance of these forms and spaces, as well as the functional, formal and symbolic roles they play in the organization. For a form to be articulated as being important or significant to an organization, it mist be made uniquely visible. This emphasis can be achieved by endowing a form or shape with;

- exceptional size

- a unique style

- a strategic location (Ching, 1996:338) (Fig.3).

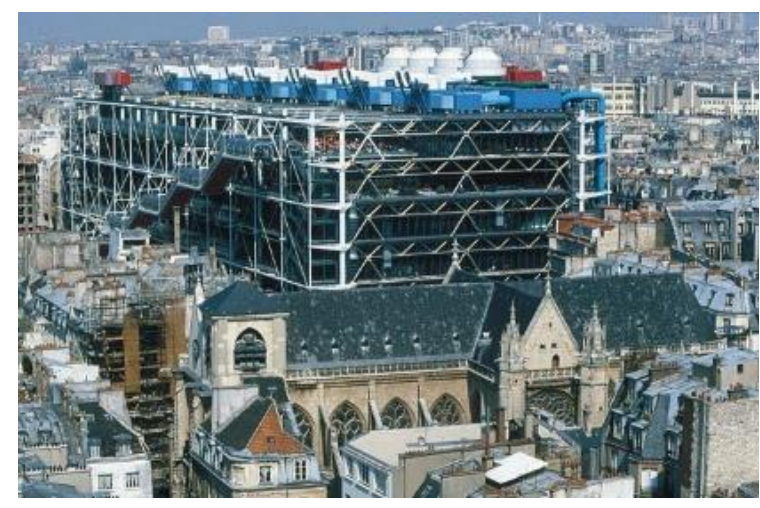

Figure 3: Centre Pompidou as a sample for hierarchy with a huge size (https://www.arkitektuel.com/centre-pompidou/)

\subsection{Analyzing values:}

(1) Formal design of building/bridge, (2) Unique style of building/bridge, (3) Technology usage in building / bridge design, (4) Sustainability issues (if exist) Icons are famous not simply for being famous, as in the case for various forms of celebrity, but famous for possessing specific symbolic/aesthetic qualities, qualities that are the subject of considerable debate within the specialist fields and, increasingly, with the recent rise of the blogosphere, debate to which the general public actively contributes (Jencks C, 2000).

When we look through architectural view, for a building/bridge to be an iconic, it must consist of a variety of integrated features such as; 'unique design, large scale, high level, spectacular representation and a specific message signify by the building'. (Jencks $C, 2000)$ And, iconicity in architecture is also defined as fame or a special symbolic/aesthetic significance that is contributed with buildings, places and sometimes architects them. An iconic design can be separated into two through their inspiration points, it can have a relationship with culture of nation or it can have a unique design in global era with consisting different type of features like technology \& material usage or sustainability (Jencks C,2000).

\subsection{General Mapping on Iconic Bridges from Past to Present}

Through sample case analyses its determined that all 15 bridges have iconic design criteria by being too long, too high, too innovative and too aesthetic with a unique form double curve generally. The most common feature is the form and aesthetic perception that they create in the city, by a curved deck (FOOTBRIDGE MEDIACITY UK), steel tensegrity structure (KURILPA BRIDGE), diamond shape (CLYDE ARCH BRIDGE), double helix form, formal resemblance to anaconda (PYTHON BRIDGE), unique wave form (HENDERSON WAVE BRIDGE). Secondly, sample case analyses determine innovative and unique technological features such as; being too long, too high with extraordinary dimensions such as Millia Viaduct bridge by being the tallest bridge between Paris and Barcelona, or Phyton bridge by being the longest foot bridge and Golden Gate bridge by being the longest suspension bridge in the world. Thirdly, sample case analyses also determine spatial features such as; multi-functional usages in addition to linkage such as ; Ponte Vecchio bridge in Florence with 


\section{Journal of}

Design Studio

v:2 n:2 December 2020

housing units indicating a distinctive façade design with colourful windows \& balconies and Zaragoza pavilion bridge which is both museum and bridge (Table 2.).

Table.2. General mapping on iconic bridges from past to present identifying iconic features. (bridges are also classified into two parts; 'structural and functional' in the table through their including closed-spaces for other functions)

\begin{tabular}{|c|c|c|c|c|c|c|c|c|}
\hline & $\begin{array}{l}\text { Name of } \\
\text { the } \\
\text { Bridge }\end{array}$ & $\begin{array}{l}\text { Architect\& } \\
\text { date\&city }\end{array}$ & $\begin{array}{l}\text { Type of the } \\
\text { bridge }\end{array}$ & $\begin{array}{l}\text { Landmark } \\
\text { property }\end{array}$ & $\begin{array}{l}\text { Iconic } \\
\text { features }\end{array}$ & $\begin{array}{l}\text { Structur } \\
\text { e }\end{array}$ & Principles & Visual perception \\
\hline 1. & $\begin{array}{l}\text { Büyükçek } \\
\text { mece } \\
\text { bridge. } \\
\text { (structural } \\
\text { ) }\end{array}$ & $\begin{array}{l}\text { Mimar } \\
\text { sinan, } \\
1567-68, \\
\text { Istanbul. }\end{array}$ & $\begin{array}{l}\text { Pedestrian } \\
\text { and vehicle } \\
\text { bridge. }\end{array}$ & $\begin{array}{l}\text { Huge\&long } \\
: \\
\text { Crosses sea } \\
\text { and lake. }\end{array}$ & $\begin{array}{l}\text { Unique } \\
\text { form, } \\
\text { Large scale. }\end{array}$ & $\begin{array}{l}\text { A three- } \\
\text { stage } \\
\text { pointed } \\
\text { arched } \\
\text { stone } \\
\text { bridge. }\end{array}$ & $\begin{array}{c}\text { Rythm, } \\
\text { Scale\& } \\
\text { Proportion, } \\
\text { Hiearchy, } \\
\text { Symetry }\end{array}$ & 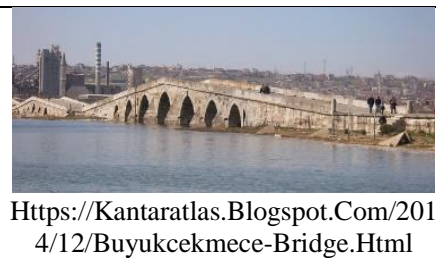 \\
\hline 2 & $\begin{array}{l}\text { Ponte } \\
\text { vecchio } \\
\text { bridge. } \\
\text { (functiona } \\
\text { 1) }\end{array}$ & $\begin{array}{l}\text { Taddeo } \\
\text { gaddinri di } \\
\text { fioravante, } \\
1345, \\
\text { Floransa }\end{array}$ & $\begin{array}{l}\text { Pedestrian } \\
\text { bridge- } \\
\text { multifuncti } \\
\text { onal } \\
\text { (stores+brid } \\
\text { ge). }\end{array}$ & $\begin{array}{l}\text { City center } \\
\text { iconic. }\end{array}$ & $\begin{array}{l}\text { Bridge } \\
\text { Façade } \\
\text { design, } \\
\text { Multi- } \\
\text { functional } \\
\text { usage. }\end{array}$ & $\begin{array}{l}\text { Stone } \\
\text { build. }\end{array}$ & $\begin{array}{c}\text { Rythm, } \\
\text { Scale\& } \\
\text { Proportion, } \\
\text { symetry }\end{array}$ & 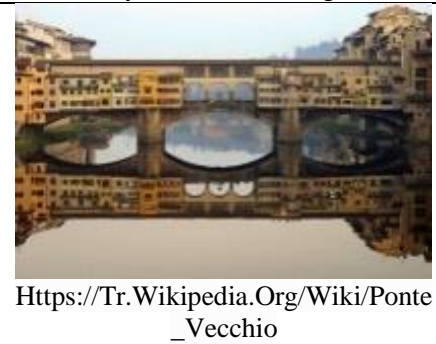 \\
\hline 3 & $\begin{array}{l}\text { Henderso } \\
\mathrm{n} \text { wave } \\
\text { bridge- } \\
\text { Functiona } \\
\text { 1. } \\
\text { (structural } \\
\text { ) }\end{array}$ & $\begin{array}{l}\text { Singapore, } \\
\text { 2008.ura }\end{array}$ & $\begin{array}{l}\text { Pedestrian } \\
\text { Public } \\
\text { spaces with } \\
\text { sittings. }\end{array}$ & $\begin{array}{l}\text { Connects } \\
\text { two hills of } \\
\text { mount } \\
\text { faber and } \\
\text { telok } \\
\text { blangah } \\
\text { hill. }\end{array}$ & $\begin{array}{l}\text {-highest } \\
\text { pedestrian } \\
\text { bridge. } \\
\text {-unique } \\
\text { wave-form. }\end{array}$ & $\begin{array}{l}\text { Wave } \\
\text { form } \\
\text { made up } \\
\text { of seven } \\
\text { undulatin } \\
\text { g curved } \\
\text { steel } \\
\text { "ribs". }\end{array}$ & $\begin{array}{c}\text { Rythm, } \\
\text { Scale\&prop } \\
\text { ortion, } \\
\text { Hiearchy }\end{array}$ & $\begin{array}{l}\text { Https://En.Wikiarquitectura.Com/Buil } \\
\text { ding/Henderson-Wave-Bridge/ }\end{array}$ \\
\hline 4 & $\begin{array}{l}\text { London } \\
\text { tower } \\
\text { bridge. } \\
\text { (structural } \\
\text { ) }\end{array}$ & $\begin{array}{l}\text { River } \\
\text { thames, } \\
1894, \text { by } \\
\text { john wolfe } \\
\text { barry and } \\
\text { horace } \\
\text { jones. }\end{array}$ & $\begin{array}{l}\text { The bascule } \\
\text { bridges- } \\
\text { brickwork } \\
\text { in a feudal } \\
\text { style of } \\
\text { arch.. }\end{array}$ & $\begin{array}{l}\text { The } \\
\text { landmark } \\
\text { its place in } \\
\text { history of } \\
\text { the river } \\
\text { thames. }\end{array}$ & $\begin{array}{l}\text { The piers of } \\
\text { the tower } \\
\text { bridge are } \\
\text { different } \\
\text { from an } \\
\text { ordinary } \\
\text { bridge. } \\
\text { 1982- the } \\
\text { glass } \\
\text { walkway. }\end{array}$ & $\begin{array}{l}\text { Stone } \\
\text { towers } \\
\text { and cable } \\
\text { stayed. }\end{array}$ & $\begin{array}{c}\text { Scale\&prop } \\
\text { ortion, } \\
\text { Hiearchy, } \\
\text { Symetry }\end{array}$ & $\begin{array}{l}\text { Https:/En.Wikipedia.Org/Wiki/To } \\
\text { wer_Bridge }\end{array}$ \\
\hline 5 & $\begin{array}{l}\text { Sydney } \\
\text { harbour } \\
\text { bridge. } \\
\text { (structural } \\
\text { ) }\end{array}$ & $\begin{array}{l}\text { John } \\
\text { bradfield, } \\
\text { march 19th } \\
\text { 1932,sydne } \\
\text { y. }\end{array}$ & $\begin{array}{l}\text { Rail-road, } \\
\text { bicycle and } \\
\text { pedestrian- } \\
\text { traffic. }\end{array}$ & $\begin{array}{l}\text { Iconic, } \\
\text { Port } \\
\text { jackson } \\
\text { (sydney } \\
\text { harbor) }\end{array}$ & $\begin{array}{l}\text { Large scale, } \\
\text { Longest. }\end{array}$ & $\begin{array}{l}\text { Steel } \\
\text { Through } \\
\text { arch } \\
\text { bridge. }\end{array}$ & $\begin{array}{c}\text { Scale\&prop } \\
\text { ortion, } \\
\text { Hiearchy }\end{array}$ & $\begin{array}{l}\text { Https://Www.Ice.Org.Uk/What-Is- } \\
\text { Civil-Engineering/What-Do-Civil- } \\
\text { Engineers-Do/Sydney-Harbour- } \\
\text { Bridge }\end{array}$ \\
\hline
\end{tabular}

Journal of Design Studio, v:2 n:2

Arslan, A., (2020), Bridges as City Landmarks: A Critical Review on Iconic Structures, 
Journal of

Design Studio

v:2 n:2 December 2020

\begin{tabular}{|c|c|c|c|c|c|c|c|c|}
\hline 6 & $\begin{array}{l}\text { Golden } \\
\text { Gate } \\
\text { bridge. } \\
\text { (structural } \\
\text { ) }\end{array}$ & $\begin{array}{l}1937, \\
\text { strauss, san } \\
\text { francisco. }\end{array}$ & $\begin{array}{l}\text { Rail-road, } \\
\text { bicycle and } \\
\text { pedestrian } \\
\text { traffic. }\end{array}$ & $\begin{array}{l}\text { Between } \\
\text { san } \\
\text { francisco } \\
\text { bay and } \\
\text { the } \\
\text { pacific } \\
\text { ocean. }\end{array}$ & $\begin{array}{l}\text { Longest } \\
\text { suspension } \\
\text { bridge in the } \\
\text { world. } \\
\text { Art nouve } \\
\text { elements } \\
\text { Color usage. }\end{array}$ & $\begin{array}{l}\text { Steel, } \\
\text { A } \\
\text { suspensio } \\
\text { n bridge. }\end{array}$ & $\begin{array}{c}\text { Scale\&prop } \\
\text { ortion, } \\
\text { Hiearchy }\end{array}$ & Https://En.Wikipedia.Org/Wiki/Golde \\
\hline 7 & $\begin{array}{l}\text { Millia } \\
\text { viaduct } \\
\text { bridge. } \\
\text { (structural } \\
\text { ) }\end{array}$ & $\begin{array}{l}\text { Foster\&part } \\
\text { ners } 1996 .\end{array}$ & $\begin{array}{l}\text { Highway } \\
\text { rail-road } \\
\text { bridge } \\
\text { Innovative } \\
\text { Contempor } \\
\text { ary. }\end{array}$ & $\begin{array}{l}\text { Valey of } \\
\text { the river } \\
\text { tarn }\end{array}$ & $\begin{array}{l}\text { The tallest } \\
\text { bridge in the } \\
\text { world } \\
\text { between paris } \\
\text { and barcelona }\end{array}$ & $\begin{array}{l}\text { Pylons } \\
\text { and } \\
\text { abutment } \\
\text { s }\end{array}$ & $\begin{array}{c}\text { Rythm, } \\
\text { Scale\&prop } \\
\text { ortion, } \\
\text { Hiearchy }\end{array}$ & $\begin{array}{c}\text { Hitt///Www.Highestbridges.Com/Wi } \\
\text { kindex.Php\%3Ftitle\%3dmillau_Viad } \\
\text { ut }\end{array}$ \\
\hline 8 & $\begin{array}{l}\text { Python } \\
\text { bridge. } \\
\text { (structural } \\
\text { ) }\end{array}$ & $\begin{array}{l}\text { West } 8 \\
\text { design, } \\
\text { Amsterdam } \\
1998\end{array}$ & $\begin{array}{l}\text { Pedestrian } \\
\text { bridge- } \\
\text { innovative }\end{array}$ & $\begin{array}{l}\text { Spans the } \\
\text { canal of } \\
\text { sporenbu } \\
\text { rg-borneo } \\
\text { island. }\end{array}$ & $\begin{array}{l}\text { Longest foot } \\
\text { bridge. } \\
\text {-formal } \\
\text { resemblence: } \\
\text { Anaconda } \\
\text {-innovative }\end{array}$ & Steel. & $\begin{array}{c}\text { Rythm, } \\
\text { Scale\&prop } \\
\text { ortion, } \\
\text { Hiearchy }\end{array}$ & 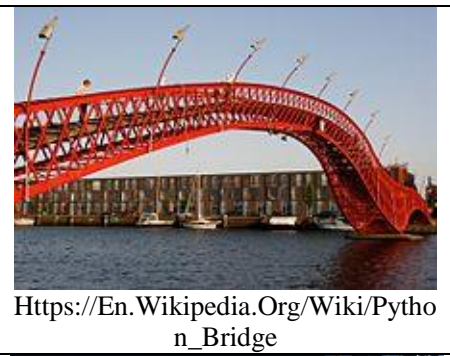 \\
\hline 9 & $\begin{array}{l}\text { Helix } \\
\text { bridge. } \\
\text { (structural } \\
\text { ) }\end{array}$ & $\begin{array}{l}\text { Cox group } \\
\text { pte ltd } \\
\text { (australia) } \\
\text { and arup- } \\
24 \text { april } \\
2004 \text { - } \\
\text { singapore. }\end{array}$ & $\begin{array}{l}\text { Pedestrian } \\
\text { bridge- } \\
\text { innovative } \\
\text { \&high-tech. }\end{array}$ & $\begin{array}{l}\text { Crosses } \\
\text { singapore } \\
\text { river. }\end{array}$ & $\begin{array}{l}\text { Unique style: } \\
\text { the world's } \\
\text { first "double- } \\
\text { helix" } \\
\text {-unique form }\end{array}$ & $\begin{array}{l}\text { Frittered } \\
\text {-glass } \\
\text { and } \\
\text { perforate } \\
\text { dstainless } \\
\text { steel }\end{array}$ & $\begin{array}{c}\text { Rythm, } \\
\text { Scale\&prop } \\
\text { ortıon, }\end{array}$ & $\begin{array}{l}*^{*} \\
\text { Https://Www.Archdaily.Com/185400/ } \\
\text { Helix-Bridge-Cox-Architecture-With- } \\
\text { Architect }\end{array}$ \\
\hline 10 & $\begin{array}{l}\text { Hangzhou } \\
\text { bay } \\
\text { bridge. } \\
\text { (structural } \\
\text { ) }\end{array}$ & $\begin{array}{l}14 \text { june } \\
2007 \text {,china, }\end{array}$ & $\begin{array}{l}\text { Highway } \\
\text { rail-road } \\
\text { bridge } \\
\text { Innovative }\end{array}$ & $\begin{array}{l}\text { Connects } \\
\text { the } \\
\text { municipa } \\
\text { lities of } \\
\text { jiaxing } \\
\text { and } \\
\text { ningbo in } \\
\text { zhejiang } \\
\text { province. }\end{array}$ & $\begin{array}{l}\text { Large scale: } \\
\text { longest trans- } \\
\text { oceanic } \\
\text { bridges in the } \\
\text { world. } 22 \\
\text { miles long. }\end{array}$ & $\begin{array}{l}\text { Cable } \\
\text { stayed } \\
\text { bridge }\end{array}$ & $\begin{array}{l}\text { Rythm, } \\
\text { Hiearchy }\end{array}$ & 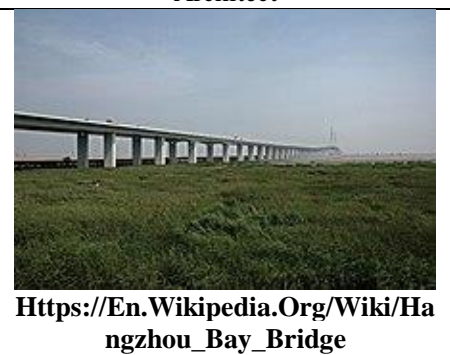 \\
\hline 11 & $\begin{array}{l}\text { Zaragoza } \\
\text { pavillon } \\
\text { bridge- } \\
\text { (functiona } \\
\text { l) }\end{array}$ & $\begin{array}{l}\text { Zaha hadid, } \\
\text { Zaragoza } \\
\text { spain,2008 }\end{array}$ & $\begin{array}{l}\text { Pedestrian } \\
\text { bridge } \\
\text { Multifuctio } \\
\text { nal: } \\
\text { Pavillon } \\
\text { bridge }\end{array}$ & $\begin{array}{l}\text { Enclosed } \\
\text { space } \\
\text { spanning } \\
\text { the river } \\
\text { ebro to } \\
\text { the } \\
\text { zaragoza } \\
\text { expo } \\
2008\end{array}$ & $\begin{array}{l}\text { Unusual } \\
\text { typology: a } \\
\text { union of two } \\
\text { building } \\
\text { typologies }\end{array}$ & $\begin{array}{l}\text { 4 pods- } \\
\text { steel-high } \\
\text { tech } \\
\text { materials }\end{array}$ & $\begin{array}{c}\text { Rythm, } \\
\text { Scale\&prop } \\
\text { ortion, } \\
\text { Hiearchy }\end{array}$ & $\begin{array}{c}\text { Https://Www.Dezeen.Com/2008/06/ } \\
\text { 16/Zaragoza-Bridge-Pavilion-By- } \\
\text { Zaha-Hadid/ }\end{array}$ \\
\hline
\end{tabular}

Journal of Design Studio, v:2 n:2

Arslan, A., (2020), Bridges as City Landmarks: A Critical Review on Iconic Structures, 
Journal of

Design Studio

v:2 n:2 December 2020

\begin{tabular}{|c|c|c|c|c|c|c|c|c|}
\hline 12 & $\begin{array}{l}\text { Borough } \\
\text { high } \\
\text { street } \\
\text { bridge. } \\
\text { (functiona } \\
\text { 1) }\end{array}$ & $\begin{array}{l}\text { Jestico+ } \\
\text { Whiles } \\
\text { London }\end{array}$ & $\begin{array}{l}\text { Pedestrian } \\
\text { bridge-tube } \\
\text { bridge }\end{array}$ & $\begin{array}{l}\text { Located in } \\
\mathrm{a} \\
\text { conservatio } \\
\mathrm{n} \text { area and } \\
\text { the busy } \\
\text { borough } \\
\text { market }\end{array}$ & $\begin{array}{l}\text { The } \\
\text { iconic } \\
\text { feature } \\
\text { bridge is } \\
70 \mathrm{~m} \\
\text { long, } 9 \mathrm{~m} \\
\text { high in } \\
\text { the centre }\end{array}$ & $\begin{array}{l}\text { Steel- } \\
\text { bridge is } \\
70 \mathrm{~m} \text { long, } \\
9 \mathrm{~m} \text { high in } \\
\text { the centre }\end{array}$ & $\begin{array}{l}\text { Scale\&prop } \\
\text { ortion, } \\
\text { Hiearchy }\end{array}$ & $\begin{array}{l}\text { Https:/Structurae.Net/En/Structur } \\
\text { es/Borough-High-Street-Bridge }\end{array}$ \\
\hline 13 & $\begin{array}{l}\text { Clyde } \\
\text { arch } \\
\text { bridge. } \\
\text { (structural } \\
\text { ) }\end{array}$ & $\begin{array}{l}\text { Glasgow, } \\
\text { uk } \\
\text { Gillespies } \\
\text { architect, } \\
2006\end{array}$ & $\begin{array}{l}\text { Road } \\
\text { bridge, } \\
\text { Rapid } \\
\text { transit tram } \\
\text { system and } \\
\text { four lanes } \\
\text { of traffic. }\end{array}$ & $\begin{array}{l}\text { Crosses the } \\
\text { river cydle } \\
\text { in glasgow, } \\
\text { for access } \\
\text { to pacific } \\
\text { quay area }\end{array}$ & $\begin{array}{l}\text { The } \\
\text { diamond } \\
\text { shaped } \\
\text { arch } \\
\text { section is } \\
\text { a unique. }\end{array}$ & $\begin{array}{l}96 \text { m.span } \\
\text { single arch } \\
\text { rib and } \\
\text { hangers }\end{array}$ & $\begin{array}{c}\text { Rythm, } \\
\text { Scale\&prop } \\
\text { ortion, } \\
\text { Hiearchy }\end{array}$ & $\begin{array}{c}\text { Https://Structurae.Net/En/Structur } \\
\text { es/Clyde-Arc }\end{array}$ \\
\hline 14 & $\begin{array}{l}\text { The } \\
\text { footbridg } \\
\text { e } \\
\text { mediacity } \\
\text { uk- } \\
\text { (structural } \\
\text { ) }\end{array}$ & $\begin{array}{l}\text { Wilkinson } \\
\text { eyre } \\
\text { architects, } 2 \\
012\end{array}$ & Pedestrian & $\begin{array}{l}\text { Spans the } \\
\text { manchester } \\
\text { ship canal }\end{array}$ & $\begin{array}{l}\text { Unique } \\
\text { by the } \\
\text { deck is a } \\
\text { curved } \\
\text { both in } \\
\text { plan and } \\
\text { elevation }\end{array}$ & $\begin{array}{l}\text { Asymmetri } \\
\text { c cable- } \\
\text { stayed steel } \\
\text { swing } \\
\text { bridge }\end{array}$ & $\begin{array}{c}\text { Rythm, } \\
\text { Scale\&prop } \\
\text { ortion, } \\
\text { Hiearchy }\end{array}$ & $\begin{array}{l}\text { Https://Structurae.Net/En/Structur } \\
\text { es/Media-City-Footbridge }\end{array}$ \\
\hline 15 & $\begin{array}{l}\text { Kurilpa } \\
\text { bridge, } \\
\text { australia } \\
\text { (structural } \\
\text { ) }\end{array}$ & $\begin{array}{l}\text { Payner } \\
\text { architects, } \\
\text { brisbane, } \\
2009\end{array}$ & $\begin{array}{l}\text { Pedestrian } \\
\text { and cycle }\end{array}$ & $\begin{array}{l}\text { Crosses } \\
\text { brisbahe } \\
\text { river }\end{array}$ & $\begin{array}{l}\text { Form of } \\
\text { public } \\
\text { space- } \\
\text { Unique } \\
\text { structure }\end{array}$ & $\begin{array}{l}\text { Steel } \\
\text { tensegrity } \\
\text { Structure }\end{array}$ & $\begin{array}{c}\text { Rythm, } \\
\text { Scale\&prop } \\
\text { ortion, } \\
\text { Hiearchy }\end{array}$ & $\begin{array}{l}\text { Htps://Www.Archdaily.Com/186214/ } \\
\text { Kurilpa-Bridge-Cox-Rayner- } \\
\text { Architects-With-Arup }\end{array}$ \\
\hline & $\begin{array}{l}\text { Name of } \\
\text { the } \\
\text { Bridge }\end{array}$ & $\begin{array}{l}\text { Architect\& } \\
\text { date\&city }\end{array}$ & $\begin{array}{l}\text { Type of the } \\
\text { bridge }\end{array}$ & $\begin{array}{l}\text { Landmark } \\
\text { property }\end{array}$ & $\begin{array}{l}\text { Iconic } \\
\text { features }\end{array}$ & Structure & Principles & Visual perception \\
\hline
\end{tabular}

Journal of Design Studio, v:2 n:2

Arslan, A., (2020), Bridges as City Landmarks: A Critical Review on Iconic Structures, 


\section{Revealing Bridges Landmark Features with Two Themes: Structural Diversity \& Functional Diversity}

This part investigates selected bridges through their spatial properties and classifies into two; a) structural diversity which introduces bridges with innovative construction techniques that create sculptural structures such as; stone, suspended steel or cable-stayed steel construction and b) functional diversity introduces multi-functional bridges with additional functions such as museum bridge that create building bridges.

\subsection{Structural Diversity: Büyükçekmece Bridge \& Golden Gate Bridge \& Millau Viaduct}

\subsubsection{Sinan's Büyükçekmece bridge}

Sinan, who is one the most talented master architect of the period, had produced some cluster of bridges and aqueducts in addition to platforms horizantally. (Fig.4) In order to distribute the weight and avoid overloading, Sinan had designed large number of arches to each span. (Necipoğlu G,2005) In addition, Sözen explained the structure of stone made bridge as; 'By building slenderer piers, expanding the span of the arches, raising the road level of the bridge and replacing the triangular cross-section of the structure, architect created the stability essential to its long survival' (Necipoğlu G, 2005).

Turgut Cansever, on the other hand, describes the bridge as a structure between the sea and the lake, surrounding the lake and inside the core of the soft topography, interconnection of low heighted four peaks. The bridge which landmarks its environment with its calm sharp, geometrical lines and forms, resembles like the white sparkling waves of Marmara Sea (Cansever, 2005).
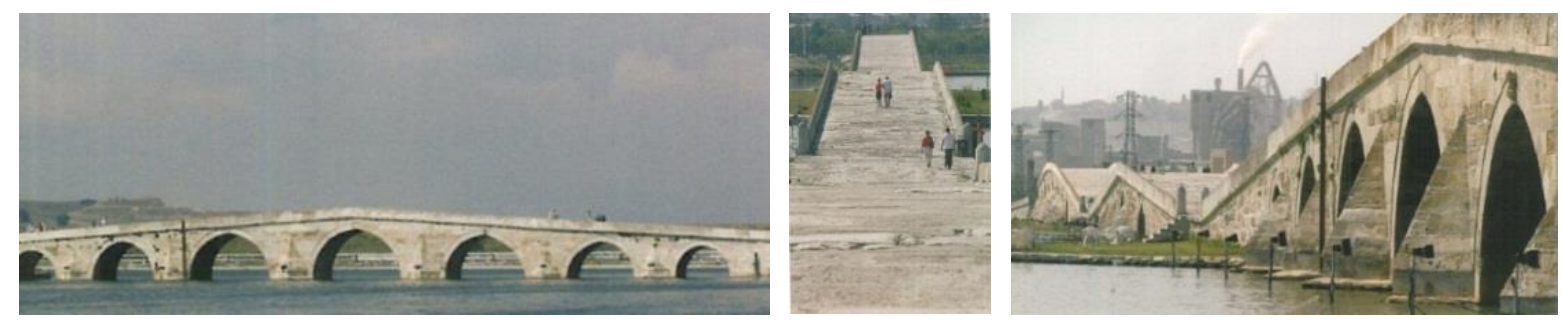

Figure.4. Views from Büyükçekmece bridge, (Necipoğlu G,2005)

other projects which can be seen as architectural superstructures of its own period.

Design of the bridge: Sinan had constructed Büyükçekmece Bridge within a concept of interlinked four bridges consisting series of spans and lanchet arches that covers a total length of 635 meters. The bridge has had to be a bit long due to its place which forms a landmark concept between the lake and the sea. Master architect Sinan solved the length problem by developing a repetitive structural model concept with integrated aesthetic values such as; totatly four interlinked bridges that transfer the major stresses with the equilateral triangular members which support the

\section{A. Aesthetic\&Artistic analyses of the bridge:} This part includes principle analyses of the bridge design

a. Rhythm principle: Reveals between spans, arches and four bridges. At bridge, a mathematical repetition of same elements and forms can be seen. The continuity of architectural elements such as lancet arches and slender spans with different sizes, creates an aesthetic rhythm. (Fig.5)

\section{b. Color, texture, light principle of the} bridge: The texture and color of stone material create a unique harmony and unity with its time and environment. 


\section{c. Proportion \& Order \& From Principle:} Proportions of each peak and arches in-between create a human-scale form.

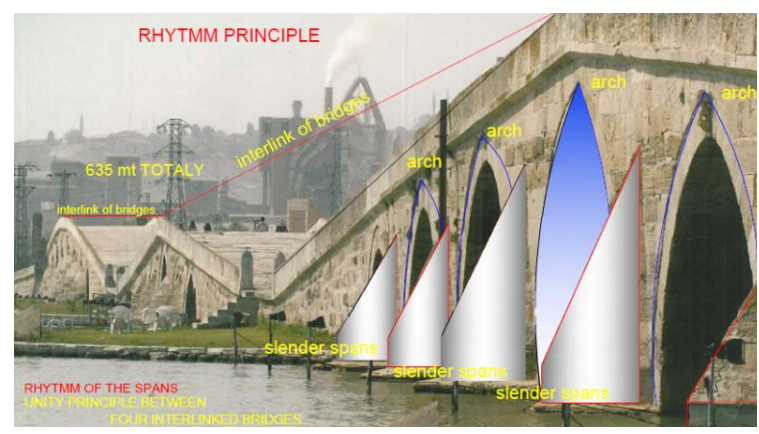

Figure.5. Rhythm principle analyses of the bridge. Rhythm, between interlinked bridges, arches and spans and how they create an architectural aesthetics value. (Necipoğlu G,2005)

\section{d. Hierarchy by organization of rythmic} arcitectural elements; by placing two same size units both side of a smaller unit, variety appears by the use of architectural instruments such as, arches, slender, spans. and so on. Emphasis principle is very visible by the peaks designed from beginning to the end like waves.

\section{B. Landmark Value analyses}

a. Formal design of Büyükçekmece Bridge: The Bridge resembles big waves within more geometric shapes like triangles which indicate the formal design approach of the architect. In addition, bridge resembles the mountains behind formally and gets in harmony with them by an urban spatial imagination. b. Unique style of the bridge: The Bridge's unique style comes from its form and long distance it crosses with making peaks for three times.

c. Technology usage: Through the construction date of bridge, there weren't existing technological devices. It is a traditional designed bridge with vernacular materials such as stone usage, latchet arches and slender spans. d. Sustainability issues: The Bridge consists of sustainable elements such as vernacular materials and construction methods which keep it standing till today friendly with its environment.

\subsubsection{Golden Gate Steel Suspension Bridge} The Golden Gate Bridge was used to be known as longest suspension bridge in the world till 1964 with a main span which is 1300 meters. Now its famous span length has been passed by ten different bridges over the world by the help of developing technology. Golden Gate Bridge is now the second longest bridge in the United States after Verrazano-Narrow Bridge in New York. Today Golden Gate length from abutment to abutment is totally 2.337 meters. The height analyses of the bridge is also excellent such as; the highest water average is 67 meters and while the two towers at each side reaches a height of 227 meter above water which ensures the suspension design of the bridge. They were the tallest in the world till 1998 when at Denmark and Japan higher were designed. (Fig.6)

(http://goldengate.org/exhibits)

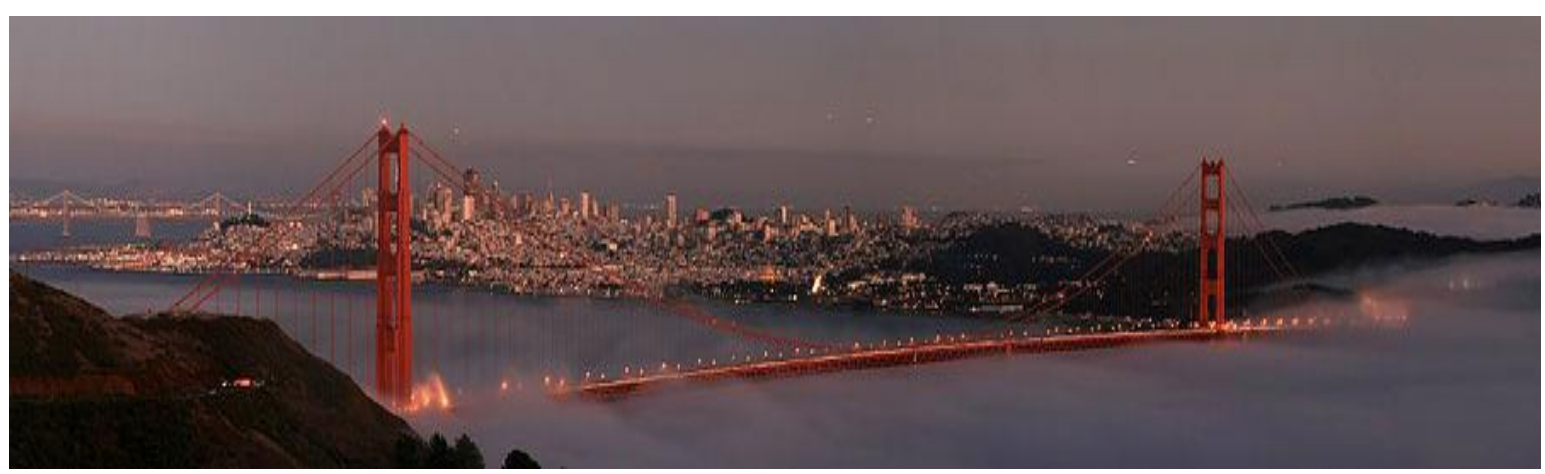

Figure.6. San Francisco with two bridges (Western section of the Bay Bridge in the left background), Coit Tower (in background to the left of North tower), and Fort Mason (on the San Francisco waterfront in the background behind the North tower) from Marin. (source:http://goldengate.org/exhibits) 
Design of the bridge: At first stages of the bridge design, Strauss was working for the overall design and construction project of the
Texture and color; by its orange color bridge really creates a harmony with its natural environment which is known as a foggy place. (Fig.7)

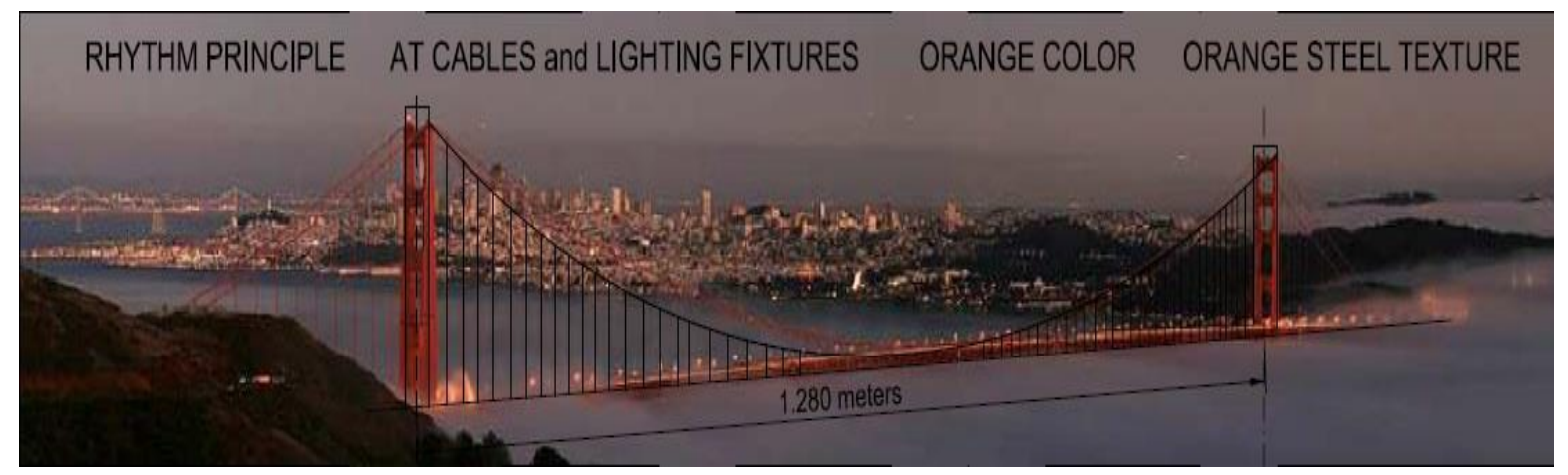

Figure.7. Aesthetic analyses of Golden GateBridge(source:http://goldengate.org/exhibits)

bridge but then due to the requirements for more experienced constructions such as; cablesuspension systems, it's decided to come over a final scheme with both architect and engineer corporation work. So Leon Moisseiff, who had experienced on suspension design with New York's Manhattan Bridge work, had been chosen for structure designer of the Golden Gate Bridge.

Steel usage \& protection: The Bridge's main body/roadway is hung by two cables from each tower symmetrically and is fixed in concrete at each end where cables reach land. Each cable is made of 27.572 strands of wire. There are 80,000 miles $(130,000 \mathrm{~km})$ of wire in the main cables.

Aesthetics: Irwing Morrow was responsible from the aesthetics values of the bridge as an architect and especially he used color at bridge for perception. Morrow choose an orange vermillion color, called international orange because it fits with natural surrounding and environment on one side and on the other side it makes the bridge perception visible in foggy atmospheres.(http://goldengate.org/exhibits)

\section{A. Aesthetic Analyses of Golden Gate \\ Bridge}

Rhythm principle; can be easily found on the iconic bridge firstly at repetition of cables and secondly at the lights of the bridge that are placed on both sides and main cables. (Fig.7)
Hierarchy by sizes: Bridge crosses huge length 1280 meters and the towers highest in the world 227meters

B. Landmark Feautures: Golden Gate bridge is a landmark in the city San Fransicso by including iconic features such as first longest suspension bridge in the world with a railroad span of 2.337 meters long connecting San Francisco Bay and Pacific Ocean. Although it's a big structure, the bridge is in a harmony with its environment like a sunset with its orange colour, its visibility in the fog and its thick and tall towers with art deco elements, looks like futuristic skyscrapers of both sides, like gates entering SanFransico one side, and the Marin gate on the other side.

\subsubsection{MILLAU VIADUCT, FRANCE 1993 /} cable-stayed steel bridge

Millau Viaduct Bridge constructed in France, between 1993-2004 is the longest high way bridge by 2.48 kilometers, from Paris to Barcelona, crossing the Tarn River. The bridge is an infrastructure which successfully achieves to articulate the challenge of spanning the 2.48 kilometers form one plateau to the tower in the most economical and aesthetic manner. The iconic bridge is located in southern France forms a link between A75 auto route from 
Clermont Ferrand to Beziers across the Massif Central. (Fig.8) (www.fosterandpartners.com)

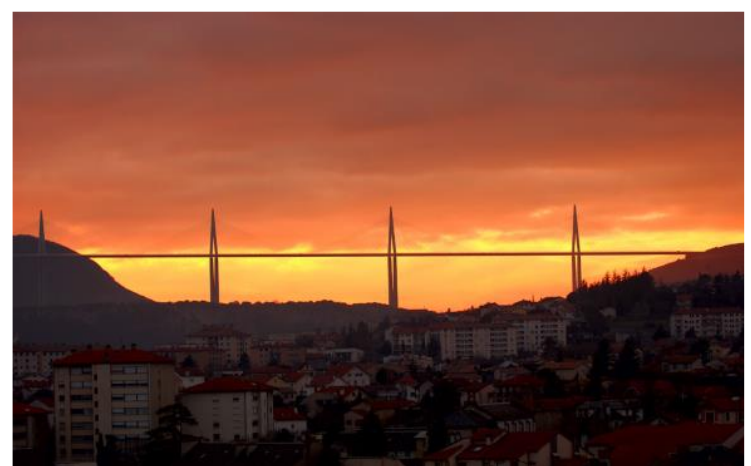

Figure.8.Millau Viaduct, France, 1993-2004) (www.fosterandpartners.com)

Design: The master structure has a cable-stayed steel construction solution, very delicate, transparent in materials and owns optimum span between the columns. The bridge is iconic by several records that it consists such as the highest pylons in the world, the highest road bridge deck in Europe, and it is the second super ceded, tallest, steel structure in France after Eiffel tower. (Fig.9-10)

(www.fosterandpartners.com)

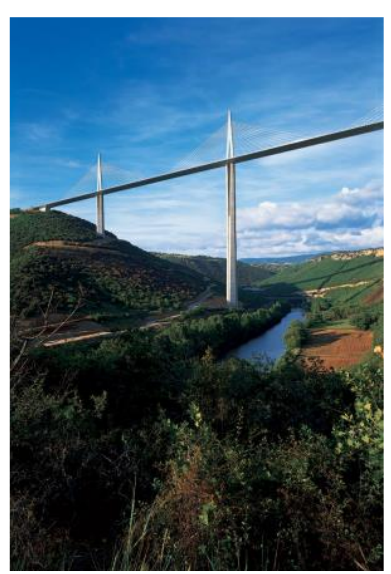

Figure 9. Environmental view of the bridge, Figure 10. Transparent elements of the bridge.

(www.fosterandpartners.com)

A.Aesthetic Analyses of Millau Viaduct: In the bridge design; repetition, rhythm, form and proportion design principles can be found.

Hierarchy by sizes: the huge length of the bridge and height creates a strong perception in the area.
B.Landmark features: Millau Viaduct is an iconic viaduct by its superoir and aesthetic

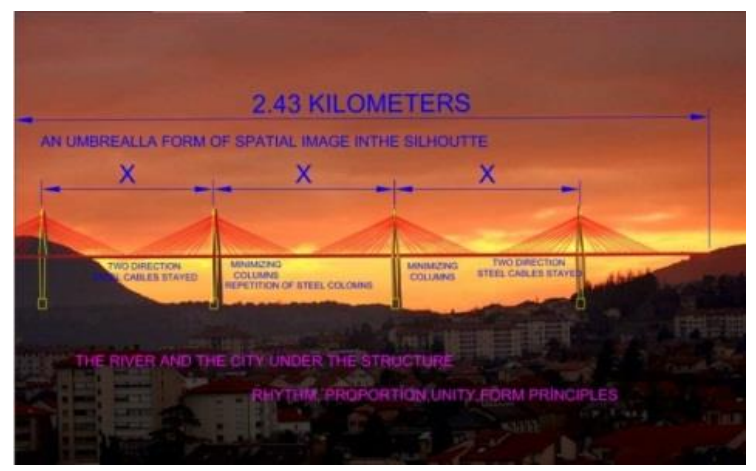

Figure.11.Aesthetic analyses of the viaduct

features such as the highest raodway bridge deck in Europe today, second tallest steel transparent struture in France after Eiffel tower. Bridge creates an excellent aesthetic silhoutte from a topografic and environmental point of view.

\subsection{Functional Diversity of Bridges}

\subsubsection{Zaha Hadid's Zaragoza Bridge} Pavillon:'Space-Frame Steel Structure' pods

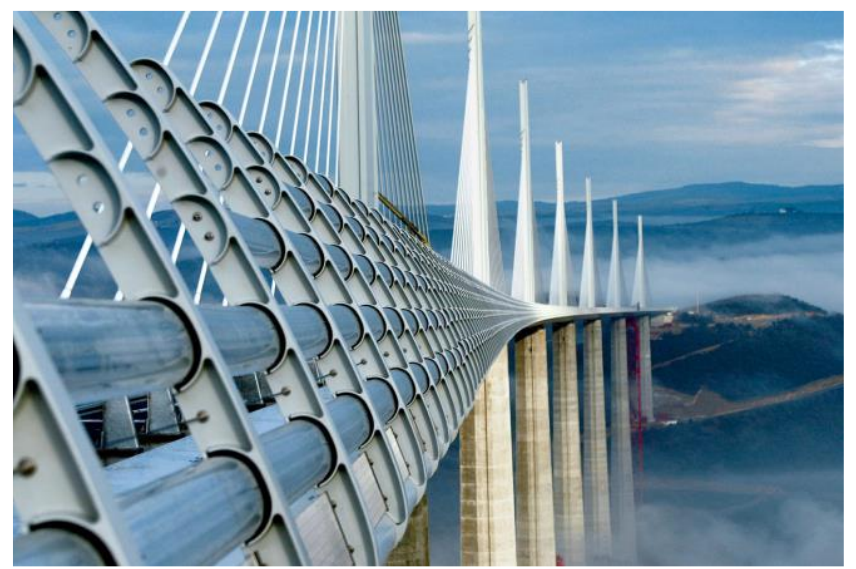

Description of the bridge: Zaragoza Bridge is a pavillon bridge with an iconic concept, placed at Zaragoza Spain, constructed between 20052008 with a size of $6.415 \mathrm{~m} 2$. The Pavillon Bridge is a multi-functional and mix use, pedestrian bridge including both exhibition spaces and shops, crosses the Ebro River.

Journal of Design Studio, v:2 n:2 
Firstly, the bridge pavilion is very unique and distinct from other bridges by its' structural design which is a steel space frame structure that acts like trusses on one hand and transfer all the pedestrian load to the floor. It's a space frame structure-bridge that creates different spatiality at each pods, four in the number totally. Each of the pods act as both interior spaces for exhibitions and it's space frame steel structures that carry the bridge. As being a landmark in the city, Pavilion Bridge has a diamond shape section and constructed and extruded around a curved path. (http://www.zaha-hadid.com/design/zaragozabridge-pavilion/)

Materials: At surface design, shark scales system is fascinating both for visual appearance and for performance. In addition, this pattern can easily wrap around complex curvatures with a simple system of rectilinear ridges. The exterior or outer skin of the pavilion is divided into two elements; the lower deck is made of structural metal plates, and the higher level consists of a cladding system of a glassreinforced concrete (GRC) panels in various shades form white to black. (Fig.12-13) (http://www.zaha-hadid.com/design/zaragozabridge-pavilion/)

Construction Description: The foundation piles of the bridge Pavillon are the deepest ever constructed in Spain with 68.5 meters. Almost 62.500 steel structure elements have been prefabricated in nine metal-fabrication workshops and subsequently assembled on site.

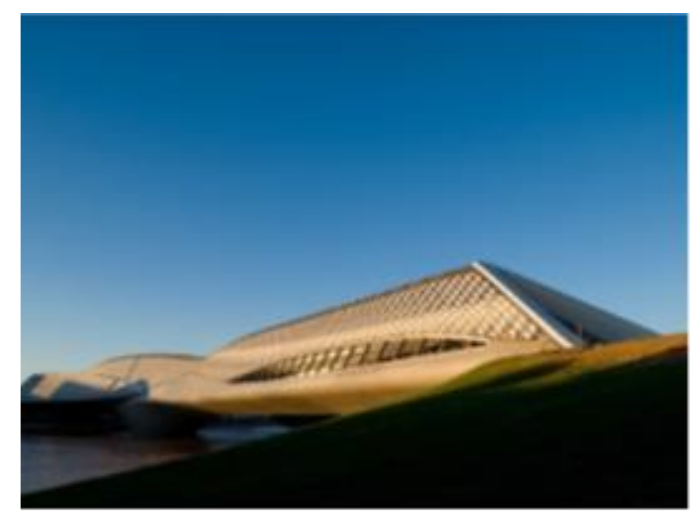

Figure.12. Exteriors views of the bridge-pavillon, (http://www.zaha-hadid.com/design/zaragoza-bridge-pavilion/)
Aesthetic values: Repetition of pods creates a rhythmic sense and by using diverse sizes at pods variety principle appears. Bridge suits with a harmony by its environmental scale and height, and has a texture value by the innovative materials that used both at interiors and exteriors. And Landmark values appear by unique construction method called; 'space frame' system at steel bridge Pavilion.

\section{Conclusions}

In the paper, it's aimed to demonstrate the aesthetic values of bridges which transforms them first icons then landmarks of their cities. With the help of general mapping on bridges, firstly, diverse functional usages of bridges have been revealed such as; pedestrian, bikepath, rail-road, tram, and even multi-functional building-bridges like pavilion integrated with exhibition spaces and shops in it. Than secondly, structural diversities have been analyzed and revealed such as; steel, cablestayed, space-frame and stone, indicating the innovative construction styles of bridges that create sculptural forms. For deep case analyses, the study is limited with four important bridge analyses which all have iconic and landmark features.

A building and/or structure can be an icon by its design or its designer/architect can be an icon too, and all four cases in the paper are icons also their designers are icons too. This is the first common feature of all selected bridges that makes them landmarks. Architect Sinan,

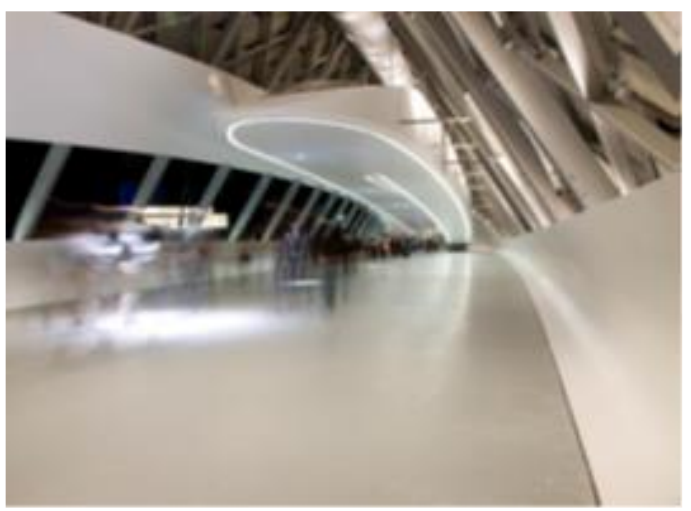

Figure.13.Interior views of the bridge-pavillon

Journal of Design Studio, v:2 n:2

Arslan, A., (2020), Bridges as City Landmarks: A Critical Review on Iconic Structures, 
Norman Foster, and Zaha Hadid; all of them are icon too and even known as star architects today. And their designs create landmarks in the city; Büyükçekmece Bridge, Golden Gate Bridge, Millau Viaduct, Zaragoza Bridge, and all of them have iconic features; Büyükçekmece Bridge by its huge length, its place crossing between the sea and lake, its' material harmony and its' geometric wave formal approach.

Secondly Golden Gate bridge, by its very long span which is the first in the world, cable-stayed steel construction with its orange color steel towers and by unique form which is delicate and aesthetics of its proportions. Thirdly, Millau Viaduct, by its height which is unique, passes over the town, with its high-tech steel constructions and material usages like transparent façade instruments. And lastly, Zaragoza Bridge, with its unique construction style, space frame steel construction, with its innovative material usage, multifunctional spaces as being both pedestrian bridge and exhibition center too (building-bridge), and its unique form creates a landmark theme. These are all iconic features that transform these bridges to landmarks of their region and city. To sum up, in the paper it's aimed to reveal the landmark features of bridges with some selected themes such as; by high-tech steel construction styles, innovative material usages, aesthetic formal approaches, multi-functional usage features and by their architects whom are icons too.

\section{References:}

Buchanan R., Doordan D., Margolin V.(2010), The Designed World:Images, Objects, Environments', Hirst J, 'Values in Design: 'Existenzminimum, Maximum Quality and Optimal Balance, Berg, Oxford, NewYork, pg.306

Cansever T. (2005), Mimar Sinan, Albaraka Türk Yayınları: 24, Kültür Kitapları: 2, ISBN:975-00582-0-8 (pages 267-273)
Ching, F. (1996); Architecture: Form, Space and Order, John Wiley and Sons.

Morris, C.W., (1939), Esthetics and the Theory of Signs, Journal of Unified Science, VIII p.131

Morris, C.W., (1948), The Open Self , New York, p.55.

Morris C.W. (1946), Signs, Languages and Behavior, New York, p.195.

Jencks, C., (2000), Iconic Building, Rizzoli International Publications.

Inns, T., (2007). Designing for the 21th Century-Interdisciplinary Question and Insights, 'Spatial Imagination in Design, Gower Publishing, England

Kaymakcan, M., (2006).'The Basic Design Elements and Principles Education and Applications at Graduate Degree Art Education', Master's Thesis, Dokuz Eylul University, Education Sciences Institute,Fine Arts Education Department.

Necipoğlu, G. (2005). The Age of Sinan Architectural Culture in the Ottoman Empire, Reaktion Books. (Sinan: Architect of Ages' (the Republic of Turkey, Ministry of Culture),Text by: Metin Sözen, Photographed by Sami Güner, Coordinator Mehmet Özel, (pages 218-225)

\section{References of Pictures}

http://goldengate.org/exhibits/exhibitarea1c.ph p

http://www.zaha-hadid.com/design/zaragozabridge-pavilion/

http://www.fosterandpartners.com https://kantaratlas.blogspot.com/2014/12/buyu kcekmece-bridge.html https://tr.wikipedia.org/wiki/Ponte_Vecchio https://en.wikiarquitectura.com/building/hende rson-wave-bridge/

https://en.wikipedia.org/wiki/Tower_Bridge

https://www.ice.org.uk/what-is-civilengineering/what-do-civil-engineersdo/sydney-harbour-bridge 
https://en.wikipedia.org/wiki/Golden_Gate_Bri

dge

http://www.highestbridges.com/wiki/index.php \%3Ftitle\%3DMillau Viadut

https://en.wikipedia.org/wiki/Python Bridge

https://www.archdaily.com/185400/helix-

bridge-cox-architecture-with-architect

https://en.wikipedia.org/wiki/Hangzhou_Bay_

Bridge

https://www.dezeen.com/2008/06/16/zaragoza-

bridge-pavilion-by-zaha-hadid/

https://structurae.net/en/structures/borough-

high-street-bridge

https://structurae.net/en/structures/clyde-arc

https://structurae.net/en/structures/media-city-

footbridge

https://www.archdaily.com/186214/kurilpa-

bridge-cox-rayner-architects-with-arup 